\title{
Stability of Metastable Vacua in Gauge Mediated SUSY Breaking Models with Ultra Light Gravitino
}

\author{
Junji Hisano, Minoru Nagai, Masato Senami and Shohei Sugiyama \\ Institute for Cosmic Ray Research, \\ University of Tokyo, Kashiwa 277-8582, Japan
}

\begin{abstract}
Recently Murayama and Nomura proposed a simple scheme to construct the gauge mediation models, using metastable supersymmetry breaking vacua. It has a possibility to predict the ultra light gravitino mass $m_{3 / 2} \lesssim 16 \mathrm{eV}$, while such a light gravitino may destabilize the metastable vacua. We investigate stability of the metastable vacuum of their model. The transition rate from the false vacuum to true ones is evaluated by numerical calculation, including the Coleman-Weinberg potential destabilizing the metastable vacuum. It is found that when the messenger sector is minimal, stability of the metastable vacuum imposes an upperbound on squark mass $M_{\tilde{q}}$ for the ultra light gravitino as $M_{\tilde{q}} \lesssim 1800 \mathrm{GeV}$ at most. Squarks with this mass may be found in the LHC experiments.
\end{abstract}




\section{Introduction}

Introduction of Supersymmetry (SUSY) is one of the most attractive directions to construct models beyond the Standard Model (SM), since SUSY cures the hierarchy problem. However, when SUSY breaking mass terms of squarks and sleptons are generic in the SUSY SM, too large flavor changing neutral current (FCNC) processes are predicted. This problem is called as the SUSY flavor problem. In addition, the SUSY SM also has the SUSY CP problem. The SUSY breaking trilinear and bilinear scalar coupling terms ( $A$ and $B$ terms) and the gaugino mass terms have $\mathrm{CP}$ phases generically. The predictions for electric dipole moments are rather large, though the constraints by experiments are severe.

The Gauge Mediated SUSY Breaking (GMSB) models [1, 2] are an excellent solution to these problems. SUSY breaking in a hidden sector is transmitted to the visible sector through the SM gauge interactions, which are flavor-blind. The SUSY breaking mass terms of squarks and sleptons are then flavor-blind, so that dangerous FCNC processes are naturally suppressed. In addition, the phases of the $A$ and $B$ terms are automatically aligned to those of the gaugino mass terms in the Minimal Gauge Mediation (MGM) model [3], since the $A$ and $B$ terms are vanishing at tree level in the model. In the case, the SUSY CP problem is avoided \#1.

In the GMSB models, the lightest SUSY particle is the gravitino, with mass $m_{3 / 2} \simeq(1 \mathrm{eV}$ - $10 \mathrm{GeV})$. An ultra light gravitino $m_{3 / 2} \lesssim 16 \mathrm{eV}$ is known to have no cosmological gravitino problem, such as overclosure [5] and too large free-streaming lengths [6]. However, only a few models were previously proposed in order to realize the ultra light gravitino [7].

Recently Murayama and Nomura proposed in Ref. [8] a simple scheme to construct the GMSB models along a metastable SUSY breaking scenario. Metastable SUSY breaking is recognized as an interesting alternative SUSY breaking scenario, after Intriligator, Seiberg and Shih found that even simple vector-like theories, such as SUSY SU(N) QCD, can break SUSY on metastable local minima [9]. Many phenomenological models with metastable SUSY breaking vacua are nowadays discussed.

The scheme proposed by Murayama and Nomura has a quite simple structure and a possibility of the ultra light gravitino mass $m_{3 / 2} \lesssim 16 \mathrm{eV}$. However, the stability of the metastable vacua becomes weaker for smaller gravitino mass. It is not clear whether the lifetimes of the metastable vacua are longer than the age of the universe.

In this paper, we study stability of the SUSY breaking metastable vacuum in the GMSB model which is realized in their scheme as an effective theory [8], in the case of the ultra light gravitino mass. The lifetime of the metastable vacuum is numerically evaluated in the semiclassical techniques [10]. In the evaluation we include the Coleman-Weinberg (CW) potential in the scalar potential, which destabilizes the metastable vacuum. In Ref. [8], the authors estimated the lifetime by a triangular approximation [11. However,

\footnotetext{
${ }^{\# 1}$ It is shown in Ref. 4 that the latest experimental values of the anomalous magnetic moment of the muon and the branching ratio of $\bar{B} \rightarrow X_{s} \gamma$ favor a low messenger scale $(\sim 100 \mathrm{TeV})$ in the MGM model. This is quite consistent with the ultra light gravitino discussed in this paper.
} 
this approximation is not accurate especially in this model, since several scalar fields are relevant to the phase transition, as the authors mentioned in their text. In the case, numerical calculation is required to estimate a reliable value of the transition rate.

We also derive upperbounds on the squark mass and the cutoff scale for ultraviolet (UV) models for $m_{3 / 2} \lesssim 16 \mathrm{eV}$. The bound on the gravitino mass can be translated to those on the squark and gluino masses in this model, since the visible sector is more directly coupled with the hidden sector in this model.

This paper is organized as follows. In the next section, we review the model discussed in Ref. [8]. In Section 3, we discuss the stability of the metastable vacuum and constraints on parameters of this model. Section 4 is devoted to summary.

\section{Model}

In this section we review the GMSB model with the metastable vacuum discussed in Ref. [8]. This model is realized in their scheme to construct the GMSB model, and has a quite simple structure. The relation between the gravitino mass and the stability of the metastable SUSY breaking vacuum is also clarified here.

The superpotential and Kähler potential in this model are given by

$$
\begin{gathered}
W=-\mu^{2} S+\kappa S f \bar{f}-M f \bar{f} \\
K=|f|^{2}+|\bar{f}|^{2}+|S|^{2}-\frac{|S|^{4}}{4 \Lambda^{2}}+\mathcal{O}\left(\frac{|S|^{6}}{\Lambda^{4}}\right),
\end{gathered}
$$

where $S$ is a gauge singlet chiral field and $f$ and $\bar{f}$ are messenger fields, which transform as 5 and $5^{*}$ under the $\mathrm{SU}(5)_{\text {GUT }}$ gauge symmetry, respectively. Here, $\mu, \kappa$ and $M$ can be taken to be real and positive without loss of generality, which are taken as free parameters in this model.

This model is an effective theory below the UV cutoff scale $\Lambda$. It is assumed that higher terms of $|S|$ appear after some particles with cutoff-scale mass are integrated out. The negative sign of the $|S|^{4}$ term is required for deriving a local minimum near the origin. The expansion in the above Kähler potential implies that $S$ has some approximate conserved charge. For example, we can assign $U(1)_{R}$ charges of 2,0 and 0 to $S, f$ and $\bar{f}$, respectively, although the last term of Eq. (11) violates this symmetry explicitly.

The tree-level scalar potential is given from Eqs. (11) and (2) as

$$
V_{\text {tree }}=\left|\mu^{2}-\kappa f \bar{f}\right|^{2}\left[1+\frac{|S|^{2}}{\Lambda^{2}}+\mathcal{O}\left(\frac{|S|^{4}}{\Lambda^{4}}\right)\right]+|\kappa S-M|^{2}\left(|f|^{2}+|\bar{f}|^{2}\right)+\sum_{i} \frac{1}{2} g_{i}^{2} D_{i}^{2} \text {. }
$$

Here, we use the same notation for scalar fields as superfields, and $D_{i}$ is the $D$ term for the SM gauge interactions $i$. Let us discuss the vacuum structure in this potential in the following. 
First, this potential has global SUSY conserving minima at

$$
S=\frac{M}{\kappa}, \quad|f|=|\bar{f}|=\frac{\mu}{\kappa^{1 / 2}},
$$

where the phases of $f$ and $\bar{f}$ are constrained as $\arg (f \bar{f})=0$. In this paper, for justifying the expansion of Eq. (2) by $S / \Lambda$, we impose the following condition,

$$
\Lambda \gtrsim \frac{M}{\kappa}
$$

In the case that this condition is not satisfied, the global minimum (4) is not determined within validity of this potential and dependent on UV models, so that we could not discuss the stability of the vacuum.

Next, this potential also has a local minimum at the origin of the field space, $S=$ $f=\bar{f}=0$. SUSY is broken in this vacuum due to non-zero $F$ term, $F_{S}=\mu^{2}$. The mass squared for the scalar component of the singlet field $S$ is given by $m_{S}^{2}=\mu^{4} / \Lambda^{2}$. The stability of this metastable vacuum becomes weaker for larger $\Lambda$, since the potential for $S$ direction becomes flat.

The masses for the fermionic and scalar components of the messenger fields are given by $M_{\text {mess }}=M$ and $m_{f_{ \pm}}^{2}=M^{2} \pm \kappa \mu^{2}$, respectively, around the origin. This minimum is metastable when the following condition is satisfied,

$$
M^{2}>\kappa \mu^{2}
$$

When $M^{2}$ is close to $\kappa \mu^{2}$, the potential for the direction along $f=\bar{f}$ is flat and this metastable vacuum becomes unstable.

The spontaneous SUSY breaking is link to the $U(1)_{R}$ symmetry [12]. However, this symmetry is approximate in this model since the SUSY mass terms of messenger fields violate it. The radiative correction from messenger fields destabilizes the SUSY breaking metastable vacuum. Thus, it is essential to include the radiative correction in discussing the stability of the metastable vacuum.

The messenger fields induce the following CW effective potential,

$$
\begin{aligned}
V_{\mathrm{CW}} & \simeq \frac{\kappa^{2} \mu^{4}}{16 \pi^{2}} F\left(\frac{|M-\kappa S|^{2}}{\kappa \mu^{2}}\right) \\
& \simeq-\frac{N_{i} \kappa^{2} \mu^{4}}{16 \pi^{2}}\left[\frac{\kappa}{M}\left(S+S^{\dagger}\right)+\frac{\kappa^{2}}{2 M^{2}}\left(S^{2}+S^{\dagger 2}\right)\right],
\end{aligned}
$$

where

$$
F(x)=\frac{N_{i}}{4}\left[(1+x)^{2} \log (1+x)^{2}+(1-x)^{2} \log (1-x)^{2}-2 x^{2} \log x^{2}\right] .
$$

Here, $N_{i}$ is the number of messenger fields that propagate in the loop diagrams, and $N_{i}=5$ in our case. In the second line, the CW potential is expanded by $S$ around the origin only 
with the leading-order contribution about $\kappa \mu^{2} / M^{2}$, and constant terms are neglected. The field value of $S$ on the local minimum is shifted toward the positive direction, and $m_{S}^{2}$ and $m_{f_{ \pm}}^{2}$ become smaller. Thus, the CW potential makes the local minimum more unstable. Nevertheless, as long as $\Lambda$ is small enough to satisfy

$$
M>\frac{\kappa^{2}}{4 \pi} \Lambda
$$

the local minimum remains and exists near the origin: $\langle S\rangle \simeq 5\left(\kappa^{2} \Lambda / 4 \pi M\right)^{2} M / \kappa$. At this false vacuum, the condition (6) does not significantly change, of course, as far as $\langle S\rangle$ is small enough.

Now we discuss the relation between the gravitino mass and the stability of the metastable SUSY breaking vacuum. First we give the gravitino mass, normalized by the squark mass, in this model. The SUSY breaking is mediated to the visible sector by messenger fields. The gaugino and sfermion masses are induced at the messenger scale $\mu_{M}$ as

$$
\begin{aligned}
& M_{\tilde{g}_{i}}\left(\mu_{M}\right) \simeq N \frac{\alpha_{i}\left(\mu_{M}\right)}{4 \pi} \frac{\kappa F_{S}}{M_{\text {mess }}} \simeq N \frac{\alpha_{i}\left(\mu_{M}\right)}{4 \pi} \frac{\kappa \mu^{2}}{M} \\
& M_{\tilde{f}}^{2}\left(\mu_{M}\right) \simeq \sum_{i} 2 N C_{i}\left(\frac{\alpha_{i}\left(\mu_{M}\right)}{4 \pi}\right)^{2}\left(\frac{\kappa F_{S}}{M_{\text {mess }}}\right)^{2} \simeq \sum_{i} 2 N C_{i}\left(\frac{\alpha_{i}\left(\mu_{M}\right)}{4 \pi}\right)^{2}\left(\frac{\kappa \mu^{2}}{M}\right)^{2}
\end{aligned}
$$

where $C_{i}$ is the quadratic Casimir, $N$ is the number of the messenger pair, and $N=1$ for our minimal case. We assumed $M \gg \kappa\langle S\rangle$ and $F_{S} \simeq \mu^{2}$ in the last form. Then, the gravitino mass is given by

$$
m_{3 / 2} \simeq \frac{F_{S}}{\sqrt{3} M_{\mathrm{pl}}} \simeq 10 \mathrm{eV}\left(\frac{M_{\tilde{q}}}{2 \mathrm{TeV}}\right)^{2}\left(\frac{M}{\kappa \mu}\right)^{2},
$$

where $M_{\mathrm{pl}}=2.4 \times 10^{18} \mathrm{GeV}$ is the reduced Planck mass. Here the mass scale is normalized by the squark mass $M_{\tilde{q}}$ calculated by Eq. (11).

Small $M / \kappa \mu$ is required in Eq. (12) for the ultra light gravitino. However, the small value of $M / \kappa \mu$ may lead to the tachyonic messengers, and the large value of $\kappa$ also destabilizes the local minimum once radiative corrections are included. The constraints ([6]) and (9) lead to the lowerbound on the value of $M / \kappa \mu$ as

$$
\left(\frac{M}{\kappa \mu}\right)^{3}>\frac{1}{4 \pi} \frac{\Lambda}{\mu} .
$$

Thus, the ultra light gravitino $m_{3 / 2} \lesssim 16 \mathrm{eV}$ is marginal.

In this estimate we omitted all possible $\mathcal{O}(1)$ coefficients. The precise value of the minimum of $M / \kappa \mu$ is essential to discuss the possibility of the ultra light gravitino in this model. In next section we evaluate the decay rate of the false vacuum numerically and constrain the parameter space of this model. 


\section{Stability of metastable vacuum}

As explained in the previous section, the stability of the SUSY breaking false vacuum is weaker for smaller gravitino mass. Hence, we should carefully estimate the transition rate of the false vacuum to the true ones. It can be evaluated in the semiclassical techniques [10]. In this method, the transition rate of false vacua described by scalar fields $\phi_{i}$ is calculated by the Euclidean path integral $\int\left[d \phi_{i}\right] e^{-S_{E}\left[\phi_{i}\right]}$, which is dominated by the so-called "bounce" configuration $\phi_{i}=\bar{\phi}_{i}(x)$. This bounce is a stationary point of the Euclidean action $S_{E}\left[\phi_{i}\right]$, that is $\delta S_{E}\left[\bar{\phi}_{i}\right]=0$, and it is known to be an $O(4)$ symmetric solution [13]. Once we find the bounce solution, the transition rate per unit volume is estimated as

$$
\Gamma / V \simeq A e^{-B} \text {. }
$$

The prefactor $A$ has the fourth power of the typical scale in the potential and

$$
B=S_{E}\left[\bar{\phi}_{i}(r)\right]-S_{E}\left[\phi_{i}^{f}\right]
$$

where $\phi_{i}^{f}$ are constant configurations that continue to stay at the false vacuum.

Several complex scalar fields exist in the present model. Hence, we should estimate $S_{E}$ by finding the bounce solution in a numerical method. In the following, we explain our calculation. For simplicity, we take all fields real and $f=\bar{f}$. The $D$ term potential for messenger fields makes this configuration $|f|=|\bar{f}|$, and the potential for the direction along $f=\bar{f}$ becomes unstable when $S$ is increased. Then, defining $\phi_{1} \equiv f=\bar{f}$ and $\phi_{2} \equiv S$, we have the following $O(4)$ symmetric Euclidean action,

$$
S_{E}\left[\phi_{i}(r)\right]=2 \pi^{2} \int_{0}^{\infty} d r r^{3}\left[\sum_{i=1}^{2} \frac{k_{i}}{2}\left(\frac{d \phi_{i}}{d r}\right)^{2}+V\left(\phi_{i}\right)\right],
$$

where $k_{1}=4, k_{2}=2$ and $V\left(\phi_{i}\right)$ is the scalar potential, including the CW potential, that is the sum of Eqs. (3) and (7). Then the bounce solution $\bar{\phi}_{i}$ is given by solving the following equations of motion,

$$
k_{i} \frac{d^{2} \phi_{i}}{d r^{2}}+k_{i} \frac{3}{r} \frac{d \phi_{i}}{d r}=\frac{\partial V}{\partial \phi_{i}}\left(\phi_{i}\right)
$$

with boundary conditions,

$$
\lim _{r \rightarrow \infty} \bar{\phi}_{i}(r)=\phi_{i}^{f}, \quad \frac{d}{d r} \bar{\phi}_{i}(r)=0 .
$$

Since the prefactor $A$ is approximately given as $A \sim \mu^{4}, B \gtrsim 400$ is required to make the lifetime of the false vacuum much longer than the age of the universe.

To solve the above equations and find the bounce configuration numerically, we used the calculation method discussed in Ref. [14]. We discretized Eqs. (17) and (18) as

$$
\begin{aligned}
& k_{i} \frac{\phi_{i, j+1}-2 \phi_{i, j}+\phi_{i, j-1}}{(\Delta r)^{2}}+k_{i} \frac{\alpha-1}{(j+\Delta j) \Delta r} \frac{\phi_{i, j+1}-\phi_{i, j-1}}{2 \Delta r}=\frac{\partial V}{\partial \phi_{i}}\left(\phi_{i, j}\right), \\
& \phi_{i, n}=\phi_{i}^{f}, \quad \phi_{i, 0}=\phi_{i, 1}
\end{aligned}
$$



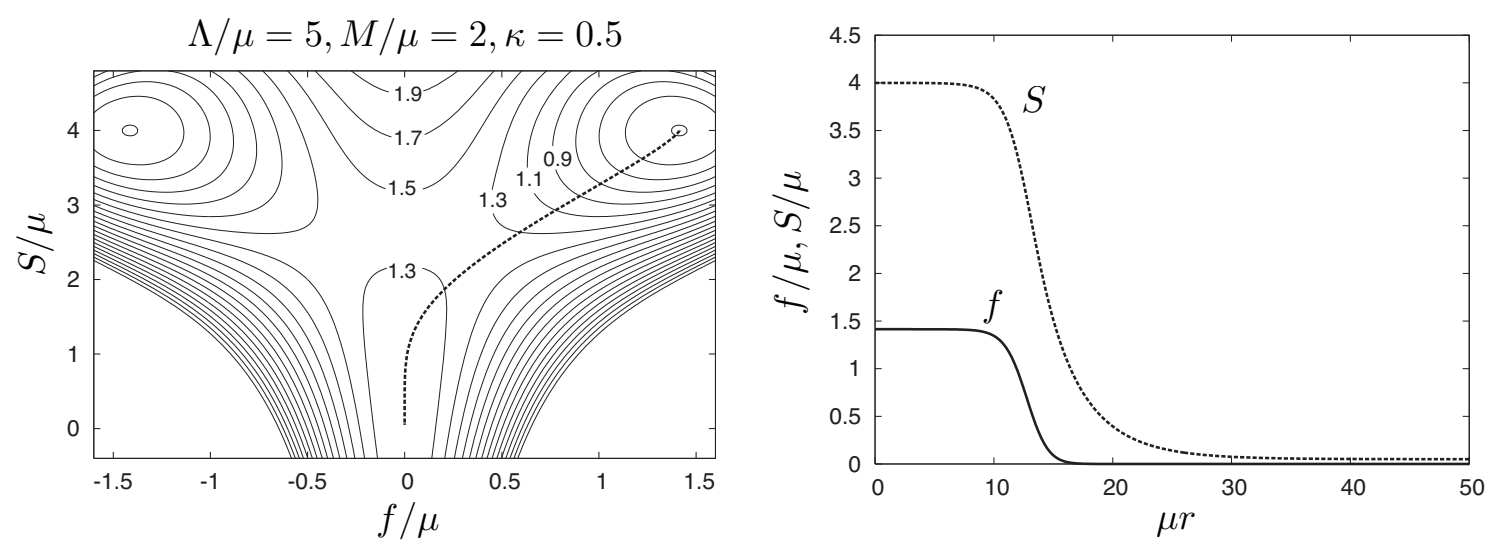

Figure 1: A typical shape of potential $V\left(\phi_{i}\right)$ (left) and dependence of bounce solution $\bar{\phi}_{i}(r)$ on $r$ (right). Parameters are taken as $\Lambda / \mu=5, M / \mu=2$ and $\kappa=0.5$. A trajectory of $\bar{\phi}_{i}(r)$ is shown by a dotted line in the left figure. Height of the potential in the figure is scaled by $\mu^{4}$.

where $\phi_{i, j}(j=0, \cdots, n)$ represents the field value of $\phi_{i}$ at the $j$-th lattice site and $\Delta r$ is the lattice size. These numerical parameters are taken to be $n=200$ and $\Delta r=0.25 / \mu$ in our calculation. Parameters $\alpha$ and $\Delta j$ in Eq. (19) are used to find the bounce configuration in this method. At first we found the undamped solution with $\alpha=1$, which can be easily obtained by an improved potential [14. Then we gradually increase $\alpha$ to 4 and decrease $\Delta j$ to 0 with solving the above equations, and finally the desired bounce solution is obtained\#2. From this bounce configuration, the coefficient $B$ is calculated by the following discretized Euclidean action,

$$
S_{E}\left[\phi_{i, j}\right]=2 \pi^{2} \sum_{j=0}^{n-1}\left(j+\frac{1}{2}\right)^{3} \Delta r^{3}\left[\sum_{i=1}^{2} \frac{k_{i}}{2}\left(\frac{\phi_{i, j+1}-\phi_{i, j}}{\Delta r}\right)^{2}+\frac{V\left(\phi_{i, j+1}\right)+V\left(\phi_{i, j}\right)}{2}\right]
$$

In Fig. 1, we show a typical potential shape (left) and the corresponding bounce configuration derived by the above procedure (right) for $\Lambda / \mu=5, M / \mu=2$ and $\kappa=0.5$. In the left figure, a trajectory between the false vacuum and true one is shown by a dotted line. In the right figure, the trajectories of $\phi_{1}(=f)$ and $\phi_{2}(=S)$ are shown by solid and dotted lines, respectively. They are rather simple in most of the parameter space as long

\#2 In addition, after one bounce solution $\phi^{*}$ is obtained for a parameter set, new bounce solutions for the new parameter sets are also iteratively derived from it. In this derivation the right-hand side of Eq. (19) is expanded as

$$
\frac{\partial V}{\partial \phi_{i}}\left(\phi_{i, j}\right) \simeq \frac{\partial V}{\partial \phi_{i}}\left(\phi_{i, j}^{*}\right)+\sum_{k=1}^{2}\left(\phi_{k, j}-\phi_{k, j}^{*}\right) \frac{\partial^{2} V}{\partial \phi_{i} \partial \phi_{k}}\left(\phi_{i, j}^{*}\right)
$$

to linearized the system of equations. 

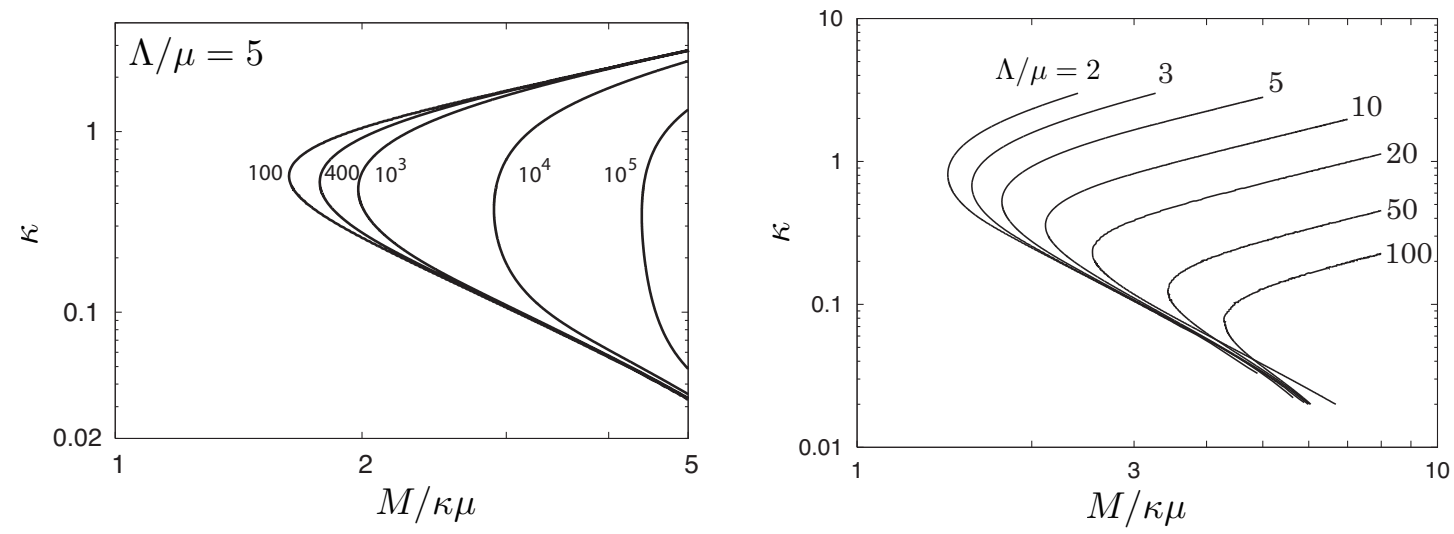

Figure 2: Values of $B$ for $\Lambda / \mu=5$ (left) are shown dependent on $M / \kappa \mu$ and $\kappa$. Constraints on the parameter spaces by $B \geq 400$ for several values of $\Lambda / \mu$ (right).

as the conditions Eqs. (5), (6) and (9) are satisfied. First $\phi_{2}$ moves toward the positive direction and $\phi_{1}$ is almost unchanged. Then around $\phi_{2} \simeq(M-\sqrt{\kappa} \mu) / \kappa$, the messenger field becomes tachyonic and both fields start to go toward one of the true vacua.

Next, we discuss the parameter dependence of the decay rate of the false vacuum. The potential $V\left(\phi_{i}\right)$ are described by 4 parameters: $\Lambda, M, \mu$, and $\kappa$. Since the overall mass scale is irrelevant to the calculation of $B$, we normalized all dimensional parameters by $\mu$. In Fig. 2 (left), we show the value of $B$ as a function of $M / \kappa \mu$ and $\kappa$ for $\Lambda / \mu=5$. In the lower-left region the vacuum is unstable along the $f(\bar{f})$ direction since the condition, $M^{2}>\kappa \mu^{2}$, in Eq. (6) is not satisfied. In the upper-left region the CW potential is not negligible and the vacuum is unstable due to the violation of the condition in Eq. (9). From this figure, it is found that $B$ is determined mainly by the value of $M / \kappa \mu$ except around the boundaries given in Eqs. (6) and (9).

In Fig. 2 (right), the allowed regions by the constraint $B \geq 400$ are shown for several values of $\Lambda / \mu$. The allowed regions are the right side of solid lines. The minimum allowed value for $M / \kappa \mu$ is almost determined by the intersecting point of the stability conditions, Eqs. (6) and (9) for fixed $\Lambda / \mu$. Hence, the bound is roughly given by Eq. (13). The difference between Eq. (13) and the bound derived by Fig. 2 is explained by two contributions. First, the numerical calculation of the transition rate cuts some region near this bound as shown in Fig. 2 (left). The other is the approximation neglecting $\mathcal{O}(1)$ factors in the calculation of Eq. (9). The rest condition (5) gives upperbound on $M / \kappa \mu$ with a constant $\Lambda / \mu$. Hence, this condition is irrelevant to the lowerbound on the gravitino mass.

The parameter dependence of $B$ is understood from an approximate estimate based on Ref. [11], where the vacuum transition rate is calculated for a triangle-shaped potential of one real scalar field. Fortunately, the trajectory of the bounce solution $\bar{\phi}_{i}(r)$ is rather simple as depicted in Fig. 1, except around the boundaries, Eqs. (6) and (9). We 

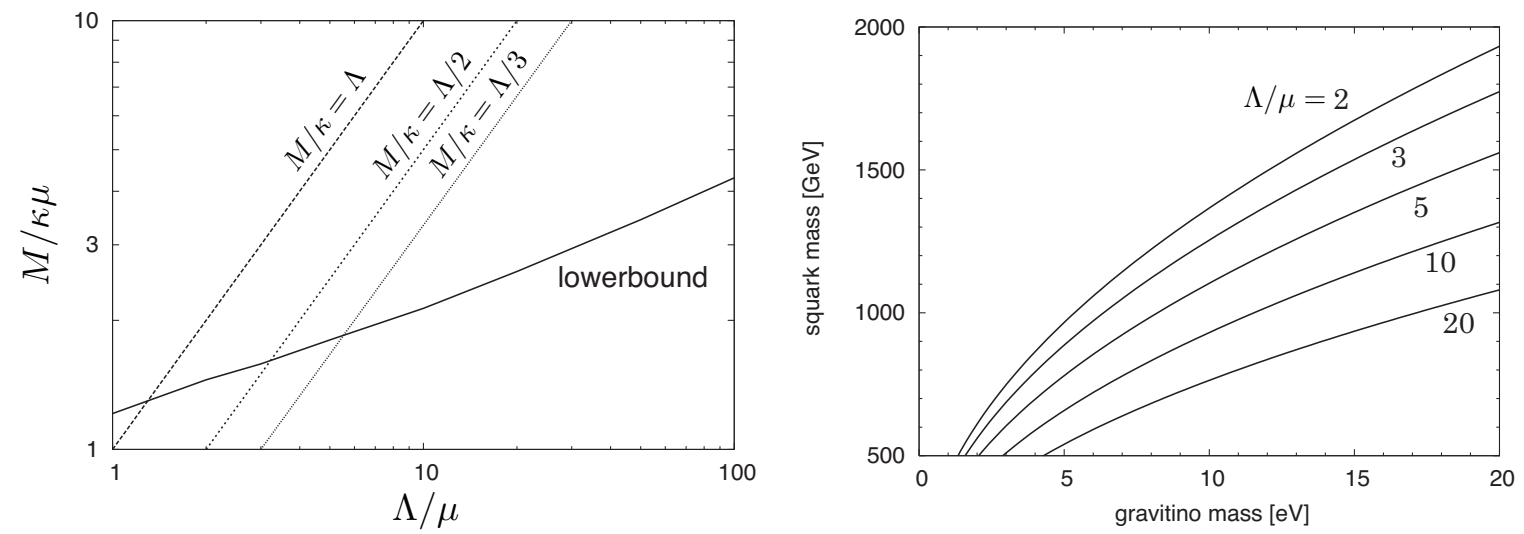

Figure 3: Constraints on $M / \kappa \mu$ as functions of $\Lambda / \mu$ (left). Solid line is lowerbound on $M / \kappa \mu$, while dotted lines show $M / \kappa \Lambda=1,1 / 2,1 / 3$ from left to right. Upperbound for the squark mass as a function of the gravitino mass (right). We take $\Lambda / \mu=2,3,5,10,20$. Allowed regions are below the lines.

approximated the potential along this trajectory by a triangle form and derived as

$$
B \simeq \frac{8 \pi^{2} M^{4}}{\kappa^{4} \mu^{4}}
$$

where this estimate is applicable for thin wall region\#3. This approximated estimate explains some features of numerical results. First, the value of $B$ depends mainly on the combination of $M / \kappa \mu$ and the power of $M / \kappa \mu$ is rather large. Second, the dependence of $B$ on $\Lambda$ is rather weak. This implies that $B$ does not change so much for different values of $\Lambda$ as long as $M / \kappa \mu$ are fixed, although the allowed region are changed.

In Fig. 3 (left), we summarized the allowed minimum values of $M / \kappa \mu$ dependent on $\Lambda / \mu$. The solid line shows the lowerbound on $M / \kappa \mu$ and the dotted lines are $M / \kappa \Lambda=$ $1,1 / 2$ and $1 / 3$, respectively. For larger values of $M / \kappa \Lambda$, the description as an effective low-energy theory becomes inadequate and higher order terms neglected in Eq. (2) become important. In these cases, UV models are needed for complete analysis.

The lowerbound on $M / \kappa \mu$ can be translated to the constraints on the gravitino and squark mass through Eq. (12). In Fig. 3 (right), we show the allowed region for the squark and gravitino masses for several values of $\Lambda / \mu$, where the allowed regions are below lines. From this figure, the upperbound on the squark mass for fixed value of $m_{3 / 2}$ is obtained. It is found that the relatively light squark mass $M_{\tilde{q}} \lesssim 1800 \mathrm{GeV}$ are required to have $m_{3 / 2} \lesssim 16 \mathrm{eV}$ even if $\Lambda / \mu=2$. When $\Lambda$ is larger, the constraint is more severe. The ultra light gravitino in this model may be checked, since the mass range is accessible in the LHC experiments. This result is derived by using the approximation of the last

\footnotetext{
\#3 Our estimate is different from that in Ref. [8], since the result in Ref. [8] can be realized using a different assumption near the boundaries.
} 
form of Eq. (12). The upperbound on the squark mass is slightly relaxed without this approximation. Nevertheless, we checked that the difference is about $10-20 \%$.

The LEP experiments constrain the SM-like Higgs boson mass as $m_{h}>114.4 \mathrm{GeV}$ [15]. For the large enough Higgs boson mass, the stop mass should be larger than about $1000 \mathrm{GeV}$ to have a large radiative correction through top and stop loop diagrams. This suggests that the cutoff scale is low enough to satisfy $\Lambda / \mu \lesssim 20$ as in Fig. 3. This is reduced to the bound, $\Lambda \lesssim 10^{7} \mathrm{GeV}$, with $\mu \simeq 200 \mathrm{TeV}\left(m_{3 / 2} / 10 \mathrm{eV}\right)^{1 / 2}$. This gives a strong implication for UV models.

Finally we briefly discuss the case of non-minimal messenger fields $(N>1)$, which is a quite interesting situation, since it may be possible to measure the gravitino mass in future collider experiments [16]. If $N$ copies of $5+\mathbf{5}^{*}$ are introduced, the gluino and squark masses become larger and scale like $N$ and $\sqrt{N}$, respectively. On the other hand, the SUSY breaking vacuum becomes more unstable, since the CW potential in Eq. (7) is proportional to $N$. A factor $N^{1 / 6}$ appears in the lowerbound on $M / \kappa \mu$ for a fixed $\Lambda / \mu$. Then the upperbounds on the gluino and squark masses scale like $N^{5 / 6}$ and $N^{1 / 3}$ respectively. Hence, squark may be still detected at the LHC experiments even for $N>1$.

\section{Summary}

In this paper we have discussed the possibility that the ultra light gravitino with $m_{3 / 2} \lesssim$ $16 \mathrm{eV}$ is realized in the GMSB model with the metastable SUSY breaking vacuum. In our numerical calculation of the decay rate of the false vacuum, the Coleman-Weinberg potential is included in the scalar potential, since it destabilizes the metastable SUSY breaking vacuum. We have found that when this model predicts a rather light gravitino, an upperbound on the squark mass $M_{\tilde{q}} \lesssim 1800 \mathrm{GeV}$ at most is obtained for the minimal messenger fields from the stability of the vacuum. They are detectable in the LHC experiments, and hence this scenario may be checked. Even for the cases of $N>1$, squarks are still relatively light. We also found that to have the ultra light gravitino the cutoff scale must be smaller than $10^{7} \mathrm{GeV}$, which is an important requirement for UV models that realize this scenario.

\section{Acknowledgments}

The works of JH and MS are supported in part by the Grant-in-Aid for Science Research, Ministry of Education, Science and Culture, Japan (No. 19034001 and No. 18034002 for JH and No. 18840011 for MS). Also, that of MN is supported in part by JSPS. 


\section{References}

[1] M. Dine, W. Fischler and M. Srednicki, Nucl. Phys. B 189, 575 (1981); S. Dimopoulos and S. Raby, Nucl. Phys. B 192, 353 (1981); M. Dine and W. Fischler, Phys. Lett. B 110, 227 (1982); C. R. Nappi and B. A. Ovrut, Phys. Lett. B 113, 175 (1982); L. Alvarez-Gaume, M. Claudson and M. B. Wise, Nucl. Phys. B 207, 96 (1982); S. Dimopoulos and S. Raby, Nucl. Phys. B 219, 479 (1982).

[2] M. Dine and A. E. Nelson, Phys. Rev. D 48, 1277 (1993); M. Dine, A. E. Nelson and Y. Shirman, Phys. Rev. D 51, 1362 (1995); M. Dine, A. E. Nelson, Y. Nir and Y. Shirman, Phys. Rev. D 53, 2658 (1996).

[3] M. Dine, Y. Nir and Y. Shirman, Phys. Rev. D 55, 1501 (1997); R. Rattazzi and U. Sarid, Nucl. Phys. B 501, 297 (1997).

[4] J. Hisano and Y. Shimizu, arXiv:0706.3145 [hep-ph].

[5] H. Pagels and J. R. Primack, Phys. Rev. Lett. 48, 223 (1982).

[6] M. Viel, J. Lesgourgues, M. G. Haehnelt, S. Matarrese and A. Riotto, Phys. Rev. D 71, 063534 (2005).

[7] K. I. Izawa, Y. Nomura, K. Tobe and T. Yanagida, Phys. Rev. D 56, 2886 (1997); K. I. Izawa, Y. Nomura and T. Yanagida, Phys. Lett. B 452, 274 (1999); K. I. Izawa and T. Yanagida, Prog. Theor. Phys. 114, 433 (2005).

[8] H. Murayama and Y. Nomura, Phys. Rev. Lett. 98, 151803 (2007); H. Murayama and Y. Nomura, Phys. Rev. D 75, 095011 (2007).

[9] K. Intriligator, N. Seiberg and D. Shih, JHEP 0604, 021 (2006).

[10] S. R. Coleman, Phys. Rev. D 15, 2929 (1977) [Erratum-ibid. D 16, 1248 (1977)]; C. G. Callan and S. R. Coleman, Phys. Rev. D 16, 1762 (1977).

[11] M. J. Duncan and L. G. Jensen, Phys. Lett. B 291, 109 (1992).

[12] A. E. Nelson and N. Seiberg, Nucl. Phys. B 416, 46 (1994).

[13] S. R. Coleman, V. Glaser and A. Martin, Commun. Math. Phys. 58, 211 (1978).

[14] T. Konstandin and S. J. Huber, JCAP 0606, 021 (2006).

[15] W. M. Yao et al. [Particle Data Group], J. Phys. G 33, 1 (2006).

[16] K. Hamaguchi, S. Shirai and T. T. Yanagida, Phys. Lett. B 651, 44 (2007). 